\title{
Moving Ahead: A New Centre of Research Excellence in Brain Recovery, Focusing on Psychosocial Reintegration Following Traumatic Brain Injury
}

\author{
Skye McDonald, ${ }^{1}$ Vicki Anderson, ${ }^{2}$ Jennie Ponsford, ${ }^{3}$ Robyn Tate, ${ }^{4}$ Leanne Togher, ${ }^{4}$ \\ Angela Morgan, ${ }^{2}$ Jennifer Fleming, ${ }^{5}$ Tamara Ownsworth, ${ }^{6}$ Jacinta Douglas, ${ }^{7}$ and \\ Bruce Murdoch ${ }^{5}$ \\ ${ }^{1}$ University of New South Wales, Sydney, Australia \\ ${ }^{2}$ Murdoch Childrens Research Institute, Melbourne, Australia \\ ${ }^{3}$ Monash University, Melbourne, Australia \\ ${ }^{4}$ University of Sydney, Sydney, Australia \\ ${ }^{5}$ University of Queensland, Brisbane, Australia \\ ${ }^{6}$ Griffith University, Brisbane, Australia \\ ${ }^{7}$ La Trobe University, Melbourne, Australia
}

\begin{abstract}
Severe traumatic brain injury (TBI) is the most common cause of brain injury in the Western world and leads to physical, cognitive and emotional deficits that reduce independence. Changes to psychosocial function are the most disruptive, resulting in vocational difficulties, family stress and deteriorating relationships, and are a major target for remediation. But rehabilitation is expensive and its evidence base is limited. Thus, new collaborative initiatives are needed. This article details the development of 'Moving Ahead', a model for a Centre of Research Excellence (CRE) for Traumatic Brain Injury Rehabilitation. This CRE offers several major innovations. First, it provides an integrated, multi-faceted approach to addressing psychosocial difficulties embracing different clinical standpoints (e.g., psychological, speech pathology, occupational therapy) and levels of investigation (e.g., basic science to community function) across the lifespan. It is based upon a close relationship with clinicians to ensure transfer of research to practice and, conversely, to ensure that research is clinically meaningful. It provides an integrated platform with which to support and train new researchers in the field via scholarships, postdoctoral fellowships, websites, meetings, mentoring and across-site training, and thus build workforce capacity for individuals with TBI and their families. It has input from the international community to contextualise research more broadly and ensure scientific rigour. Finally, it provides collaboration across sites to facilitate research and data collection.
\end{abstract}

Keywords: traumatic brain injury, rehabilitation, psychosocial function

Severe traumatic brain injury (TBI) from motor vehicle crashes, assaults and falls, is the most common cause of brain injury in the Western world and will surpass many diseases as the major cause of death and disability by the year 2020 (Hyder, 2007). Ten million people are affected worldwide

Address for correspondence: Professor Skye McDonald, School of Psychology, University of NSW, Sydney, 2052, NSW, Australia.E-mail: s.mcdonald@unsw.edu.au 
annually. In the USA, 5.3 million people live with disability from TBI, with 230,000 new cases per annum and up to 90,000 surviving with severe disabilities (Thurman, 1999). In Australia, there were an estimated 1493 new cases of moderate TBI and 1000 new cases of severe TBI in 2008 (Access Economics, 2009).

Severe TBI leads to physical, cognitive and emotional deficits that reduce independence. Changes to psychosocial function are the most disruptive, resulting in vocational difficulties, family stress and deteriorating relationships (Tate, Broe, Cameron, Hodgkinson, \& Soo, 2005). The cost of poor social behaviour is profound. People with TBI have fewer employment opportunities (Doctor et al., 2005; Ponsford, Olver, Curran, \& $\mathrm{Ng}, 1995$ ), poorer quality of life (Dahlberg et al., 2006) and experience social isolation (Demakis et al., 2007). They are also at high risk of depression, which further impacts on their ability to integrate back into the community (GomezHernandez, Max, Kosier, Paradiso, \& Robinson, 1997). Deterioration in function and social isolation results in dependence on recurrent government health funding, e.g., the lifetime costs of brain injuries in Australia, in 2008 alone, were $\$$ A8.6 billion (Access Economics, 2009). With normal life expectancies and more casualties each year, the social burden is cumulative and climbing rapidly. Children with such injuries are doubly disadvantaged as they are ill-equipped to learn normal skills in the process of becoming mature, socially competent adults.

Clearly, psychosocial difficulties are a major target for remediation. But rehabilitation is astonishingly expensive and the evidence base for existing remediation techniques is limited (Cullen, Chundamala, Bayley, \& Jutai, 2007) especially for social reintegration (McCabe et al., 2007). Although intervention studies are being published at a rapid and exponential rate: e.g., 1970s $(n=5)$, 1980s $(n=47), 1990 \mathrm{~s}(n=125)$, and 2000s $(n=$ 240) (PsycBITE: www.psycbite.com), the quantity and quality of the evidence is patchy and incomplete (Cicerone et al., 2011). While interventions can be effective, there is a pressing need for research to partial out effective components of complex therapies and to focus upon relevant, functional outcomes (Cicerone et al., 2011). Lack of theoretical sophistication in remediation research is another drawback that leads to vague, nonspecific treatments (Whyte \& Hart, 2003). These issues reflect the fragmented nature of remediation research in this field and highlight the need for a coherent framework with which to guide future research and researchers. This article details the development of a Centre of Research Excellence
(CRE) for Traumatic Brain Injury Rehabilitation which has been funded by the Australian National Health and Medical Research Council (NHMRC), commencing in 2012. We have called this CRE 'Moving Ahead: a CRE in Brain Recovery' to reflect the notion that the CRE is about growth and development in terms of research, clinical practice and ultimately psychosocial outcomes for people with TBI.

We argue that a CRE in TBI Rehabilitation is urgently needed for several reasons. First, the social consequences of TBI are complex and profound. Increasing social participation requires a multi-faceted approach. Currently, individual research groups in North America, the UK and, until recently, Australia, are investigating specific issues, often taking discipline-specific approaches, with limited collaboration. The establishment of Moving Ahead with an integrated approach is a world first, providing a platform to tackle psychosocial deficits resulting from TBI on a number of co-ordinated fronts.

Second, rehabilitation for TBI requires a close relationship with clinicians to ensure translation of research into practice and to foster a culture of research in practice. What is known about psychosocial competencies after TBI and their remediation comes almost exclusively from the allied health field. Australian brain injury rehabilitation centres lead the world in terms of allied health patient care and management. Currently, there are few formal mechanisms for engaging clinicians in research and increasing their involvement in the development and implementation of cutting-edge, evidence-based therapies. Our CRE has the potential to provide a pivotal interface to translate research to clinical practice.

Third, currently there is a dearth of research career opportunities for clinicians from allied health professions, including clinical and neuropsychologists, occupational therapists, speech-language pathologists and social workers, despite their direct relevance to psychosocial disorders following TBI. Indeed, with the exception of some of the senior members of Moving Ahead, there are few health professionals in Australia working in research positions dedicated to brain injury. There are a number of obstacles that mean that once graduates have commenced clinical careers, it is challenging to attract them back to research. Generally, there are few research opportunities available. Relatedly, a major hurdle is that the field of psychosocial disorders associated with TBI is fragmented, relying upon specific individuals in separate institutions conducting independent research. This situation is not conducive to capacity building or training of clinical researchers in the multidisciplinary field 
of psychosocial rehabilitation. Moving Ahead will provide the structure and support for highly skilled clinicians to move into independent research careers via scholarships, postdoctoral fellowships, training programmes, workshops and mentoring.

Fourth, individuals with brain injury are clinically heterogeneous and dispersed in the health system, making it difficult to recruit sufficient participants for large-scale research in any single centre. Significant in-roads into TBI rehabilitation can, therefore, only be achieved through a collective effort to concentrate expertise, enable skills sharing, unify divergent approaches and facilitate data collection across sites. Finally, a CRE in TBI Rehabilitation requires the co-operation and collaboration of an interdisciplinary mix of researchers and clinicians with proven track records in research and translation.

\section{The Conceptual Framework for Moving Ahead}

\section{From the Bedside to the Barbeque}

Moving Ahead works on a model of psychosocial functioning (Beauchamp \& Anderson, 2010) which recognises that psychosocial deficits following TBI arise from a number sources: preexisting, internal factors (e.g., personality), impairment from brain damage, activity limitation due to loss of skills, and indirect effects such as anxiety and depression. Importantly, they also reflect external factors, such as social opportunities for participation which provide the context within which skills are exercised. Basic remediation research aims to ameliorate impairment of damaged neural, cognitive, emotional processes and activity limitation by training skills. These are critical components of rehabilitation. But in addition, we need to maximise relevance, motivation and social opportunities to practice new skills (Ylvisaker, Turkstra, \& Coelho, 2005) by tailoring remediation to personalised contexts. We also need to address secondary (indirect) effects such as depression and anxiety. Increasing participation, in turn, increases efficacy of remediation of impairment and skills.

\section{It is All About Timing}

Different deficits impede remediation at different points in the recovery trajectory. For example, impairments in fatigue, emotion regulation, speech and emotion perception interfere with early phase remediation. Self-awareness is especially critical for the transition back to the community. Deficits in communication and social skills may present major limitations when individuals are placed in demanding social contexts, such as work, school or with friends. Furthermore, loss of social opportunity as a result of the TBI, or failure to engage effectively with available social resources following return to the community, can deny individuals with TBI the opportunity to participate. The indirect consequences of depression and anxiety often emerge later on, when the individual is confronted with real-life feedback on impairments and limitations. Moving Ahead bases its research activities on a model for psychosocial rehabilitation that builds upon the model of Beauchamp and Anderson (2010) and which additionally operationalises transitions across time (see Figure 1 with target areas for remediation shaded). By providing remediation at the right time and place (context) and by engaging community resources, we will capitalise on brain plasticity, motivation, opportunities for learning and opportunities for success.

\section{Focus on Evidence-based Practice}

Reviews in the area of psychological interventions have consistently concluded that empirically supported models encompass: (1) empirical advances in understanding structural brain damage, plasticity and recovery (e.g., Kleim \& Jones, 2008; Robertson \& Murre, 1999); and (2) cognitive behavioural therapy (CBT) approaches (Chambless, 1993; McGinn \& Sanderson, 2001). Accordingly, the research of Moving Ahead will use these empirically supported theoretical frameworks in a coordinated research programme spanning direct remediation of neural impairments underlying psychosocial dysfunction through to the application of the latest empirically validated CBT approaches to improve social skills and psychosocial adjustment, while taking into account the need for therapy adaptations and contextual supports.

\section{Developing a Unified Research Paradigm}

As emphasised in their major review (Cicerone et al., 2011), patient characteristics, e.g., the severity of cognitive impairment and the presence of specific impairments, notably executive dysfunction, influence response to interventions and overall psychosocial outcomes. Conclusions of this and other systematic reviews repeatedly highlight the need to identify: (1) the characteristics of those who benefit from treatment; and (2) components of treatment that are effective. It is urgent that we take stock of current research directions, rather than simply proliferate treatment trials. A major function of Moving Ahead is to increase collaboration across studies in order to: (1) employ a common framework for measuring outcomes; (2) 


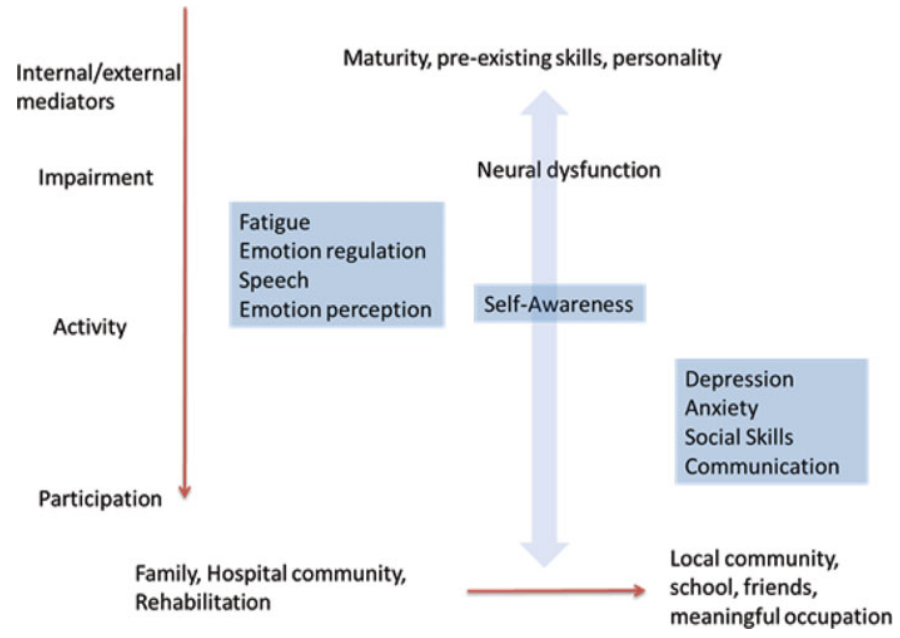

\section{FIGURE 1}

(Colour online) Model for rehabilitation emphasising transitions - from impairment to participation - from hospital to community. Different kinds of rehabilitation targets emerge at different points in these transitions and effective remediation needs to be guided by this. Domains (shaded) represent major targets for the CRE.

identify effective components of treatment by using measures specific to each component (targeting impairment, activity and participation); and (3) examine process variables, e.g., severity and nature of cognitive deficits, working alliance, selfefficacy, treatment expectations and engagement, as mediators/moderators of outcomes. This will provide a pool of data across sites and studies that can be used to identify characteristics of those who benefit from treatment generally from those who do not. The collection of data in this standardised manner will magnify the value of individual research programmes and generate opportunities for meta-analyses.

\section{Research Projects of Moving Ahead}

All of the research to be implemented in Moving Ahead addresses psychosocial deficits from basic impairment through to their effects on participation, and can be categorised as focused on either self-competency (i.e., self-regulation, fatigue, mood and self-awareness) or social competency (i.e., speech, social cognition, social skills and communication).

\section{Self-competency}

Emotional dysfunction includes acquired impairments in self-regulation of emotion due to neuropathology disrupting cortico-limbic neural circuitry (Jorge et al., 2004; Larson, Kaufman,
Schmalfuss, \& Perlstein, 2007) and secondary effects of depression, anxiety and related problems with fatigue and sleep disturbance. Collectively, these symptoms present problems for more than $60 \%$ of all TBI survivors and are associated with poorer psychosocial outcomes (Olver, Ponsford, \& Curran, 1996; Whelan-Goodinson, Ponsford, \& Schönberger, 2008). In addition, impaired selfawareness is a key barrier to successful progress in rehabilitation and is largely unaddressed in conventional rehabilitation practice (Ownsworth \& Clare, 2006). Individuals with persisting awareness deficits have long-term difficulties in regaining independence and work, and maintaining relationships (Ownsworth et al., 2007). Moving Ahead focuses upon these fundamental issues as follows.

Remediating Disorders of Self-regulation: Control and Drive

Disorders of control (e.g., anger management) are prevalent, seen in $34-67 \%$ of those with severe TBI in the first year of injury (Brooks, Campsie, Symington, Beattie, \& McKinlay, 1987; Kim, Manes, Kosier, Baruah, \& Robinson, 1999) and are maintained over time (Brooks et al., 1987; Oddy, Coughlan, Tyerman, \& Jenkins, 1985). Disorders of drive are evidenced by lowering of arousal and motivation resulting in apathy, difficulties in maintaining initiative, low spontaneity and cognitive flexibility, and are seen in $43-78 \%$ of people with severe TBI (Kant, Duffy, \& Pivovarnik, 1998; Lane-Brown \& Tate, 2009; Oddy et al., 
1985), with clinically significant degrees of apathy occurring in $15 \%$ at 18 months post-trauma (Tate et al., 2006). Traditional approaches to improving social and emotional behaviour have focused upon skills training and CBT (Alderman, 2003; Denmark \& Geneinhardt, 2002). Such techniques applied to anger management in people with TBI have demonstrated some success (Alderman, 2003; Medd \& Tate, 2000) although they may be more successful in the acute stages of recovery. Treatments to address apathy are extremely limited (Lane-Brown \& Tate, 2008). Current approaches of Moving Ahead build upon experimental research into CBT but are also investigating biofeedback as a direct remediation technique.

\section{Treatments for Fatigue, Anxiety and Depression}

Fatigue following TBI has been shown to be associated with attentional impairments, sleep disturbance and mood. Light therapy is a safe, noninvasive, non-pharmacological intervention that acts via a novel photoreceptor system mediating the circadian effects of light. Short-wavelength (blue) light has demonstrated efficacy in reducing sleepiness, enhancing reaction time and alleviating mood disturbance in healthy and patient groups. Members of the CRE based at Monash University are conducting a randomised controlled trial (RCT) to examine the effectiveness of blue light therapy versus yellow light therapy (placebo) and 'treatment as usual' on subjective daytime sleepiness and fatigue. This novel study is the first RCT of a non-pharmacological treatment for sleepiness and fatigue following TBI. If shown to be effective, Moving Ahead will use knowledge translation methods to disseminate a therapy protocol to clinicians.

Symptoms of anxiety and depression are common following brain injury, frequently persist over many years and even increase with time. Such symptoms are often identified by those with TBI and their families as particularly devastating, impacting on the capacity for participation within the community. Pharmacological interventions have limited effectiveness in the general population. There has been little research evaluating the use of psychological treatments, such as CBT, particularly in those with moderate-severe TBI, whose cognitive impairments may impede engagement in and benefit from such therapy. Our CRE is currently evaluating three approaches for reducing mood disturbance, one that is relevant for children and adolescents and two for adults.

First, we are currently conducting research using the Cool Kids programme that was developed for reducing anxiety in non-injured adolescents (Rapee et al., 2006). We have found that a modified version of this programme has relevance to children with brain injury. For example, in a single case study of an adolescent boy with TBI treated with the Cool Kids approach, we have shown that although typical questionnaire measures were insensitive to change, analysis of daily activities identified significant improvements in social participation (Soo, Tate, \& Rapee, 2012). The CRE is now linked into an RCT of the Cool Kids programme, modified for adolescents with TBI and delivered via internet and telephone as well as face to face.

Second, an NHMRC-funded RCT led by Ponsford and colleagues from Monash University, is evaluating the effectiveness of two interventions: (1) CBT alone, and (2) Motivational Interviewing (MI: a brief intervention aimed at enhancing treatment engagement and response rate to CBT) and CBT combined; in alleviating self-reported anxiety and depression in adults with TBI. MI uses a number of specific strategies to explore and resolve ambivalence to change (Miller \& Rollnick, 2002). Its efficacy has been shown both as a stand-alone treatment, and as a preparatory intervention prior to other treatment such as CBT (Burke, Dunn, Atkins, \& Phelps, 2004; Westra \& Dozois, 2006). Both CBT and MI have been adapted specifically for a community sample with moderate-severe TBI, in order to accommodate their cognitive limitations. A secondary aim is to investigate the association of cognitive factors, including IQ, memory, selfmonitoring and self-awareness, with positive response to the intervention. Results from a pilot study provide support for the hypothesised effects (Hsieh et al., 2012). Following completion of this trial, the Moving Ahead programme will provide a platform for translating these manualised interventions to clinicians around Australia.

A third psychological approach is the application of Acceptance and Commitment Therapy (ACT), including mindfulness-based approaches. ACT represents part of the third wave of behavioural therapies. It focuses upon enabling people to re-engage with meaningful life goals and has promising effectiveness in improving functionality and well-being in a variety of populations that have psychological disturbances and/or medical problems (Kangas \& McDonald, 2011). Our CRE is developing research into the application of ACT for those with TBI of varying severity.

\section{Treatment for Self-awareness}

Self-awareness is comprised of two broad skills: 'self-knowledge', or the ability to accurately perceive personal strengths and limitations; and 'online awareness', which is the capacity to selfmonitor and self-regulate behaviour during task performance (Toglia \& Kirk, 2000). Members of 
Moving Ahead have established a metacognitive intervention framework that focuses on developing self-awareness of deficits and self-regulation skills through activity participation (Ownsworth, Fleming, Desbois, Strong, \& Kuipers, 2006). This approach uses systematic feedback and graded prompts to target the following processes: (1) learning to routinely stop, check and correct errors on daily tasks; (2) reflecting on the meaning of errors on tasks to promote awareness of deficits; and (3) anticipating errors in everyday situations and planning strategy use accordingly. The optimal timing for delivery of self-awareness interventions and their impact on long-term social participation needs to be determined.

\section{Social Competency - Basic Skills}

Impaired social competence is the most distressing facet of TBI for individuals and their families (Kinsella, Packer, \& Olver, 1991). If not addressed, social isolation and dependence are inevitable. Basic abilities underpinning social competence are frequently disrupted in people with TBI. Two major facets of social competence are currently being targeted by our CRE - inability to identify emotional expressions in others (impaired in $30 \%$ or more of people with TBI) (Babbage et al., 2011; Croker \& McDonald, 2005; McDonald \& Saunders, 2005) and inability to speak clearly and accurately (dysarthria), affecting two-thirds of people with TBI) (Cahill, Murdoch, \& Theodoros, 2002).

\section{Emotion Perception}

The neuropsychological mechanisms underpinning disorders of emotion perception following TBI are not well understood. To address this, labs in our CRE based at the University of New South Wales are exploring the role of emotional responsivity and empathy in emotion recognition. Using skin conductance changes (SCR), muscle movement (measured by electromyography; EMG) and heart rate changes, we have found that poor emotion perception and empathy are common after TBI, as are reduced physiological responses (i.e., arousal and mimicry) to emotional faces (de Sousa et al., 2011; McDonald et al., 2011). However, the relationship between these disorders remains unclear. Nor do we fully understand the mechanisms underpinning impaired recognition of emotion in voice. We are, therefore, exploring very early automatic responses to facial and vocal expressions using electroencephalography (EEG) and evoked response potentials (ERP).
Speech Production

Moving Ahead is also applying novel electrophysiological and imaging methods to: (1) reveal the neurophysiological basis of dysarthria associated with TBI; (2) identify prognostic biomarkers for speech outcome; and (3) trial new intervention techniques for managing dysarthria. Specifically, collaborative work across the University of Queensland and Murdoch Childrens Research Institute (MCRI) is using EEG and cutting-edge structural (e.g., fibre tractography) and functional magnetic resonance imaging (MRI) approaches (e.g., functional connectivity analyses) to determine whether there are 'critical regions' subserving speech production that, when damaged, place an individual at heightened risk for developing speech disorder. The longer-term aim of elucidating such prognostic biomarkers is earlier identification and treatment for 'at risk' patients to optimise outcomes for those individuals. The final arm of this work is the first-ever application of Transcranial Magnetic Stimulation (TMS) for the treatment of dysarthria in adults after TBI. Preliminary work is promising, suggesting that TMS may be a successful application with long-lasting treatment effects well beyond the period of original treatment (Murdoch, Ng, \& Barwood, 2012).

\section{Social Competency - In the Community}

Psychosocial difficulties following TBI peak when individuals are faced with the complexities of returning home. Opportunities to practise and improve deficit skills are maximised when addressed in context and can be enhanced by providing appropriate supports. The following projects within the CRE examine contextual supports for socialcommunicative skills from childhood and adolescence through to late adulthood.

Facilitating Home Life

Challenging social behaviours in children postTBI are a major stress for parents. We have modified an established intervention, Signposts, a programme that utilises cognitive-behavioural principles, aimed to increase parent knowledge and competence in managing a child's challenging social difficulties. Based on pilot data, a supplementary module specific to TBI has been developed by our team at the MCRI (Woods et al., under review), and a pilot study has been completed, in which we assessed parents and children pre- and post-intervention, and at 6 and 18 months, to measure the efficacy of the interventions (Woods, Catroppa, Barnett, \& Anderson, 2011). Findings are promising, with improvements post-treatment in parent stress and mental health, as well as child 
behaviour. Importantly, these improvements have been maintained over time (Woods, Catroppa, \& Anderson, 2012).

\section{Facilitating Friendships}

Returning to study (school, technical colleges, university) and maintaining friendships following TBI is a challenging process for both the person with TBI and their peers (Mealings \& Douglas, 2010; Mealings, Douglas, \& Olver, 2012; Shorland \& Douglas, 2010). There are two approaches to dealing with this issue.

One method is to improve the communication and coping skills of the person with TBI so that they have strategies to use when faced with difficult situations. This approach has been trialled in a project with adults with severe TBI in the community, funded by the Victoria Neurotrauma Initiative led by Douglas and colleagues at La Trobe University, with promising results. This communicationspecific coping intervention programme has now been modified for use in the context of communication with friends in the return to study environment.

The second approach is to provide familiar communication partners with conversational strategies to facilitate everyday communication interactions. This approach has been the focus of a substantial body of research led by Togher. Togher and colleagues have developed a communication partner training programme entitled TBI Express which has proven successful in improving the interactions of people with TBI when talking with their families (Togher, McDonald, Tate, Power, \& Rietdijk, 2009; Togher, Power, Rietdijk, McDonald, $\&$ Tate, 2012) and paid caregivers (Behn, Togher, Power, \& Heard, in press). However, there has been a paucity of research evaluating the effectiveness of working with the friends of people with TBI as they return to work and school. The CRE is examining a two-pronged approach to deal with this: (1) teaching young people and adults with TBI coping strategies to manage communication in the context of friendship prior to return to study; and (2) providing training to friends regarding the TBI with provision of communication strategies to deal with changed communication behaviours before they return.

\section{Facilitating Meaningful Occupation}

Only $40 \%$ of adults, including older adults, return to work after severe TBI (Ponsford et al., 1995) leaving $60 \%$ with 'free' time, which, for the majority, is not spent in meaningful occupation (Tate, Lulham, Broe, Strettles, \& Pfaff, 1989), further limiting opportunities to practise/regain social skills. Moving Ahead is, therefore, conducting work to target disorders of drive to enhance efficacy of our Meaningful Occupation Program (Tate et al., 2009) to increase leisure activity.

\section{Transfer of Research to Practice}

Moving Ahead is designed, not only to conduct quality clinical research, but to ensure this translates to greater effectiveness of remediation for psychosocial impairments. As such, its charter is to engage the expertise of the broad clinical community. This has two major advantages. By involving and consulting clinicians and stakeholders in the research process we ensure that the research programme is directed towards studies identified as most important, timely and relevant for advancing current practice. Second, such involvement ensures a direct avenue for the translation of research evidence into clinical practice via clinicians, and clinical policy via key stakeholders in health and disability sectors.

Moving Ahead has five approaches to engage with the broader clinical community.

1. Clinical orientation to research. As all chief investigators of Moving Ahead are clinicianresearchers, their clinical experience pervades their research, ensuring that research questions are firmly tied to improving clinical outcomes.

2. Integration with an existing research and educational organisation. Members of Moving Ahead are office bearers, Fellows and/or members of the Australasian Society for the Study of Brain Impairment (ASSBI). ASSBI is a self-funded, not-for-profit organisation with the charter to promote education and research regarding brain impairment. By engaging ASSBI, Moving Ahead has access to a fully organised, fully funded, clinical outreach programme. ASSBI has a membership of 400 financial members comprising clinicians and researchers across disciplines, providing an expansive network of contact with clinicians across geographic regions.

3. Promotion of evidence-based databases. Members of Moving Ahead have developed two freely available databases PsycBITE (www.psycbite.com) and SpeechBITE (www.speechbite.com) (see Figure 2). These index all empirical studies that provide data on the efficacy of treatments for psychosocial, speech and other psychologically based disorders in people with brain impairment. The databases are well established (2011 hits $\approx 1,638,929)$ and used nationally $(\approx 65 \%)$ and internationally $(\approx 35 \%)$. They make a pivotal 
SpeechBITE Home page

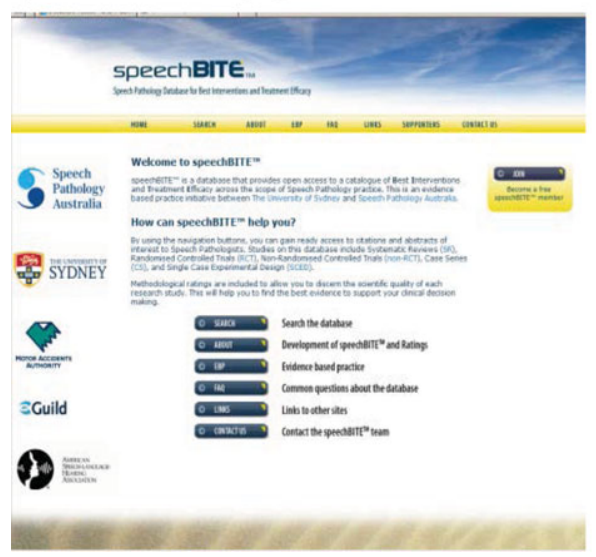

PsycBITE Home page

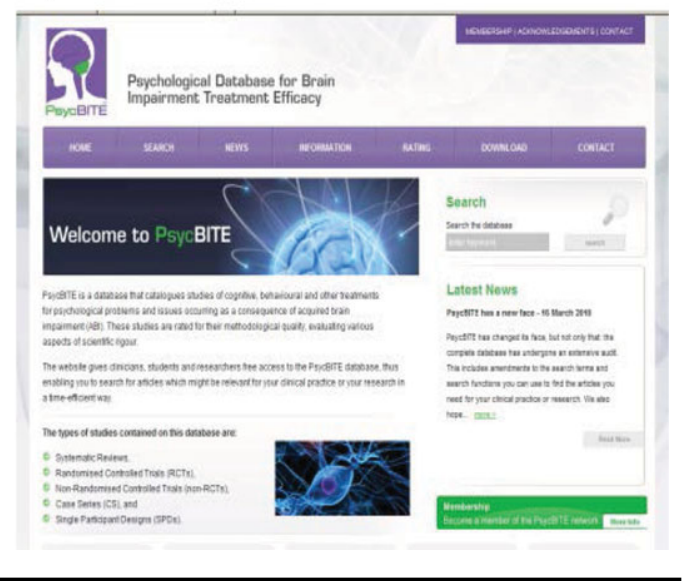

FIGURE 2

(Colour online) Home pages of PsycBITE and SpeechBITE. mechanism for transferring research outcomes into practice.

4. Partnerships with clinical centres nationally and internationally. Members of the team hold joint appointments or are affiliated with numerous clinical facilities and research institutions, providing a system of engagement with multidisciplinary clinical teams in key neuroscience and neurorehabilitation units in the most populous states of Australia (NSW, Victoria, Queensland), USA, UK, Canada and the Netherlands.

5. A formal model system for engaging with clinicians in the workplace. Moving Ahead has an established and successful model for engaging with clinicians: the Research and Evidence in Practice (REP) model (Caldwell, Whitehead, Fleming, \& Moes, 2008) which is designed to engage clinicians as consumers, participators and generators of research evidence. Our application of this model is detailed in the following section.

\section{Engaging Clinicians as Consumers}

The focus of Moving Ahead on developing a unified framework for treatment research has direct implications for clinicians and should ultimately provide real benefits for those working clinically. Most rehabilitation trials focus on treatment of specific problems in isolation. In practice, clients present with a myriad of psychological difficulties which should be treated concurrently for opti- mal outcomes. Clinical profiles of participants with TBI differ not only due to cognitive impairment but also according to levels of emotional distress, awareness of deficits and motivation (Fleming, Strong, \& Ashton, 1998). Intervention approaches need to be flexible with respect to focus and intensity. At present, there are no evidence-based guidelines to assist clinicians to match approaches with different clinical presentations. To develop guidelines there needs to be consistency in measurement across treatment trials to allow them to be collated and compared. As a world first, Moving Ahead will develop a uniform approach to treatment research by collecting common predictor and outcome measures across treatment studies, standardising occasions of outcome measurement and using measures of impairment, activity and participation specific to each treatment component. The results of this approach will assist clinicians to make informed decisions regarding what works and for whom, and will be available via the usual clinically relevant publications and conferences.

Moving Ahead has developed a website (www.moving-ahead.com.au) to interface with clinicians and researchers (see Figure 3). One of the major functions of this website is to provide access to the databases PsycBITE and SpeechBITE. Our research (Perdices et al., 2006) shows that for TBI rehabilitation, less than $30 \%$ of practitioners consult the literature to determine their choice of intervention. By integrating PsycBITE and SpeechBITE into the Moving Ahead website, along with links to sister databases PEDro (www.pedro.org.au), OTseeker 


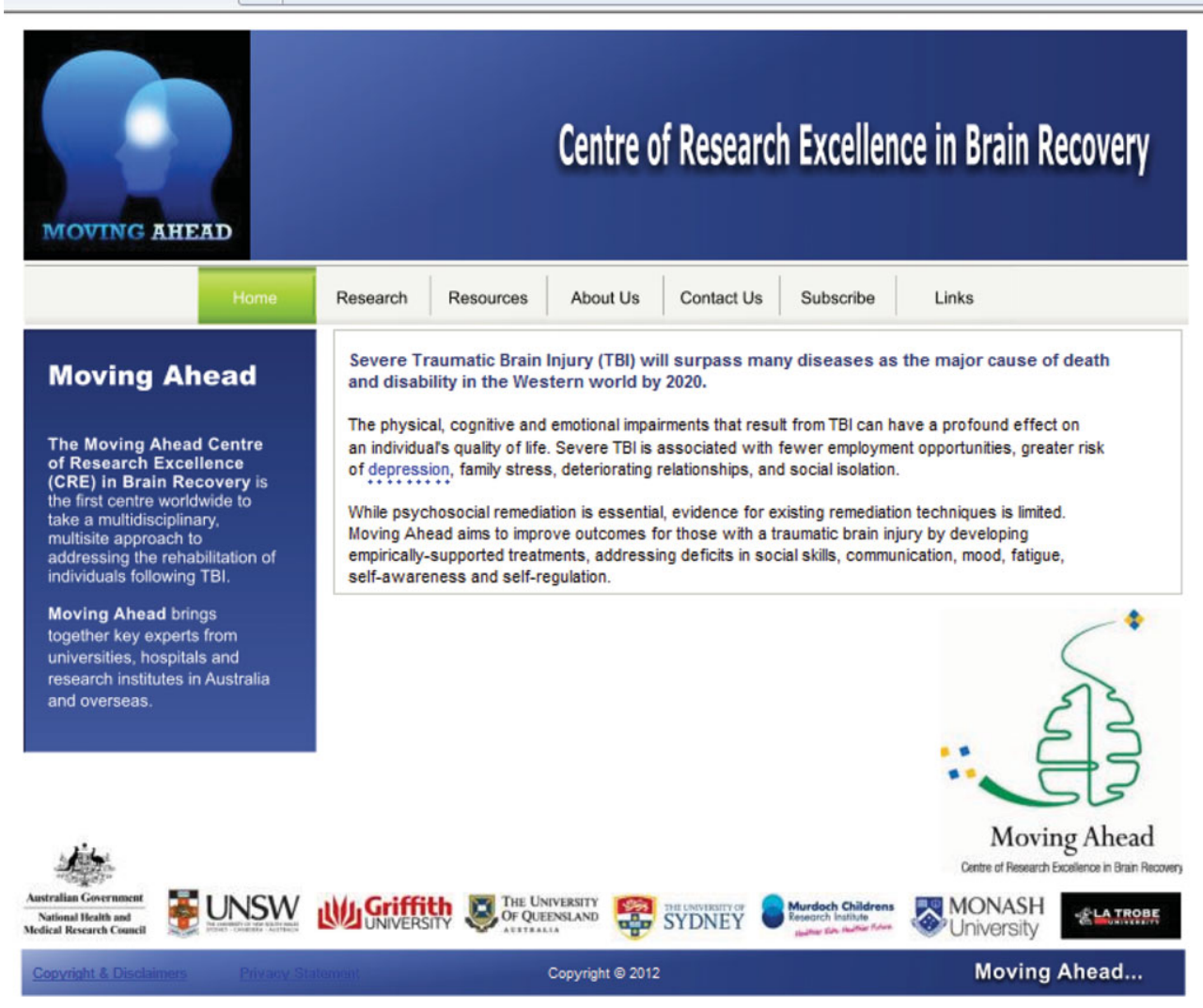

FIGURE 3

(Colour online) Home page of Moving Ahead.

(www.otseeker.com) and other TBI treatment websites such as http://www.projectlearnet.org/ we are providing an internet platform to facilitate knowledge transfer. The website also provides information regarding other activities and resources of Moving Ahead, opportunities for research participation and feedback, and links to useful websites such as advocacy and support groups.

Despite the burgeoning literature on treatments for the psychological consequences of TBI, and the expectation that clinicians will use evidence-based principles in their work, many do not know how to interpret the quality of research data or evaluate conclusions (Metcalfe et al., 2001; Young, Glasziou, \& Ward, 2002). We have developed an on-line program that provides training in how to evaluate the methodological quality of treatment trials, using step-by-step tutorials, case examples and mastery quizzes. Moving Ahead will promote and disseminate this free training (launched at the 9th Conference of the Neuropsychological Rehabilitation Special Interest Group of the World Federation of Neuro Rehabilitation (WFNR) in Bergen, July 2012) to clinicians and researchers via PsycBITE and SpeechBITE.

Another major strategy for engaging clinicians as consumers is via the publication of treatment manuals and resources. The American Psychological Association (APA) taskforce on empirically based psychological treatments insists that treatment manuals be mandatory (Chambless, 1993) but manuals informed by research rarely reach clinicians. Moving Ahead is collaborating with ASSBI Resources (http://www.assbi.com.au/assbiresources.html) to publish evidence-based treatment manuals arising from research. To date, four such manuals have been published. These are linked on the Moving Ahead website, ensuring easy access to clinicians in rural and city areas. 
Yet another initiative is to generate rehabilitation summaries. Despite APA recommendations, many evidence-based treatments in the field of TBI use experimental procedures, are based upon single-case designs, or simply lack published manuals, making it difficult for clinicians to readily access an overview of possible treatments. To address this, PsycBITE has a series of rehabilitation summaries. These summaries provide a standardised, concise, structured overview of the techniques, time frame, resources and outcome measures used in published treatment trials, along with a rating of the methodological quality of the trial. PsycBITE currently has 150 summaries of intervention for different cognitive and behavioural disorders, and it is intended that Moving Ahead will publish a set specific to evidence-based treatments for psychosocial disorders in children and adults with TBI. This will be a valuable resource for supervisors and clinicians in situations where they are unfamiliar with the current literature, treat unusual conditions, practise in remote areas or have limited access to resources. The function of rehabilitation summaries is not to replace the original article but to provide more detailed and targeted information than is available in a journal abstract, so the clinician can quickly grasp the elements of the therapy programme, the target behaviours or clinical condition that was treated, efficacy of the therapy and the methodological quality of the trial.

In addition to the 'virtual' access provided by the Moving Ahead, PsycBITE, SpeechBITE and ASSBI websites, regular face-to-face presentations are part of the platform of Moving Ahead. At a local level, continuing education workshops will play an important interface between clinician and researchers. This programme will occur within ASSBI's well-established Continuing Education programme, which organises workshops across Australia and the Pacific region, designed to meet the needs of local clinicians. Moving Ahead will also interface with annual meetings of ASSBI and other relevant societies in the region (e.g., the Australian Psychological Society College of Clinical Neuropsychologists) and further afield (e.g., the International Neuropsychological Society, the Neuropsychological Rehabilitation Special Interest Group of the WFNR, International Brain Injury Association, International Neurotrauma Society) to present symposia and workshops showcasing recent research.

\section{Engaging Clinicians as Stakeholders}

The organisational governance of Moving Ahead is designed to ensure that clinicians have direct input into the policies and research directions of the CRE. A Stakeholders Advisory Board comprises interested clinicians and/or consumer groups and provides input into whether proposed research projects meet their needs, mechanisms for translation of research findings into practice and new research directions.

\section{Engagement of Clinicians as Researchers}

A major directive of Moving Ahead is to engage clinicians as researchers, and a number of strategies are in place. For example, where appropriate, clinicians who are engaged in the collection of research data and/or delivery of experimental treatments will be affiliates of the CRE. Indeed, we already have many talented clinicians involved in existing projects. The CRE will also work closely with key clinicians to assist development of their own research directions. Support mechanisms include opportunities for a research mentoring relationship between clinicians and local members of Moving Ahead, seeding funds for new research proposals, pilot studies or development of grant applications by clinicians, part-time postdoctoral fellowships and $\mathrm{PhD}$ scholarships.

\section{Training the Researchers of Tomorrow}

Moving Ahead's strategy to build research capacity is to emulate other groups, such as medical doctors and scientists, who typically conduct research in a supported environment with large established teams (e.g., in significant public health fields such as dementia, cancer or stroke research). This provides a number of opportunities for good track record development at early career stages. Moving Ahead can provide this kind of opportunity. Further, the CRE has a number of strategies in place to attract new researchers and allied health clinicians into the field of brain injury research, including working collaboratively with clinicians and advertising via extensive university, research institute and clinical networks, and the use of its own website and that of ASSBI.

\section{Training Clinical Researchers at Different Levels of Expertise}

The strategic plan focuses on training and developing clinician-researchers who will enter the programme at different skill levels: (1) postdoctoral researchers; (2) clinical affiliates who are experienced clinicians without PhDs; (3) PhD students; (4) Masters and Honours students. Positions directly funded by Moving Ahead are advertised nationally and internationally via our institutions, networks and the Moving Ahead website. 
Additional PhD, Masters and Honours students affiliated with Moving Ahead are funded via external schemes or existing programmes. All will be encouraged to develop new research projects benefiting from the collaborative opportunities afforded by the CRE network.

Researchers in training with Moving Ahead will have opportunities to gain a variety of specialist skills that are internationally unique (e.g., speech pathologists with training in neuroscience techniques; clinical psychologists trained in psychophysiology) and trans-disciplinary (across clinical and neuropsychology, neurosciences, occupational therapy and speech pathology). They will be exposed to training in: (1) specific conceptual areas (e.g., the World Health Organisation ICF model, mechanisms and specific behavioural impacts of TBI of varying severity, common co-morbidities of TBI); (2) methodology (e.g., epidemiological design and n-of-1 trials, neuronal modelling, qualitative research approaches, use of functional magnetic resonance imaging (fMRI)); (3) practical expertise in the use of cutting-edge equipment and analysis techniques (e.g., electrophysiological methods of EEG, ERP, electromagnetic articulography; MRI data analysis techniques); and (4) research analysis and writing skills (e.g., meta-analysis and systematic reviews).

These skills will be gained via a system of training strategies. First, opportunities will be provided for short- and long-term visits by new researchers to collaborative institutions to gain hands-on training in techniques critical to their research projects. Moving Ahead will also run annual training workshops at alternating sites, the topic of training chosen to be of maximum benefit to new research team members, e.g., designing and writing competitive large-scale funding applications, development of clinical guidelines and translational research. Third, in addition to the daily supervisory support trainees receive, there will be a system of formal mentoring of postdoctoral/PhD students by members of the CRE other than their direct supervisor.

\section{Engaging with the International Research Community}

Members of the CRE have links with institutions across the USA, Canada, UK and Europe, ensuring that Moving Ahead is contextualised within the worldwide clinical research community and maximising opportunities for translation. In addition, Moving Ahead has an Expert Advisory Board consisting of internationally renowned researchers in brain recovery and rehabilitation: Pro- fessor Sureyya Dikmen, University of Washington, Seattle, USA; Professor Jonathan Evans, University of Glasgow, Scotland; Professor Tessa Hart, Moss Rehabilitation Research Institute, Philadelphia, USA; Associate Professor Mary Kennedy, University of Minnesota, USA; Professor Harvey Levin, Baylor College of Medicine, Texas, USA; Professor Brian Levine, University of Toronto, Canada; Professor James Malec, Mayo Clinic, Indianapolis, USA; Professor Lyn Turkstra, University of Wisconsin, USA; Professor Sheri Wade, University of Cincinnati, USA; Professor John Whyte, Moss Rehabilitation Research Institute, Philadelphia, USA; and Professor Barbara Wilson, Medical Research Centre, Cambridge, UK. Members of the Expert Advisory Board have been chosen to provide input on the scientific directions of Moving Ahead, especially with respect to standardising outcome measures, translation of research into practice and strengthening collaborations.

\section{Governance of the CRE}

The CRE includes key health disciplines (psychology, neuropsychology, speech pathology, occupational therapy) and is integrated with educational and clinical institutions in Australia and internationally. It has a team of Associate Investigators who will be pivotal in facilitating the translation of research into practice. The following Associate Investigators help co-ordinate CRE activities on clinical sites: Associate Professor Ian Baguley, Research Director, Westmead Brain Injury Unit, Sydney; Dr Ron Hazelton, Director, Brain Injury Rehabilitation Unit, Princess Alexandra Hospital, Queensland; Dr Clayton King, Director, Brain Injury Unit, Royal Rehabilitation Centre, Sydney; Professor John Olver, Director of Rehabilitation, Epworth Hospital, Melbourne; Associate Professor Adam Sheinberg, Director, Victorian Paediatric Rehabilitation Service, Melbourne; Dr Grahame Simpson, Research Director Liverpool Brain Injury Unit. Other associate investigators provide unique research skills to our team: Associate Professor Cathy Catroppa, MCRI, Melbourne (paediatric rehabilitation); Professor Glynda Kinsella, Latrobe University, Melbourne (older adults with TBI) and Dr Robert Heard, University of Sydney (expertise in statistical analysis).

Thus, Moving Ahead builds upon existing networks to establish a system of engagement with multidisciplinary clinical teams in key neurosciences and neurorehabilitation units nationally and internationally. Both the Expert and Stakeholder Advisory Boards will ensure that the research goals and research quality meet international standards, ensure accountability, provide 


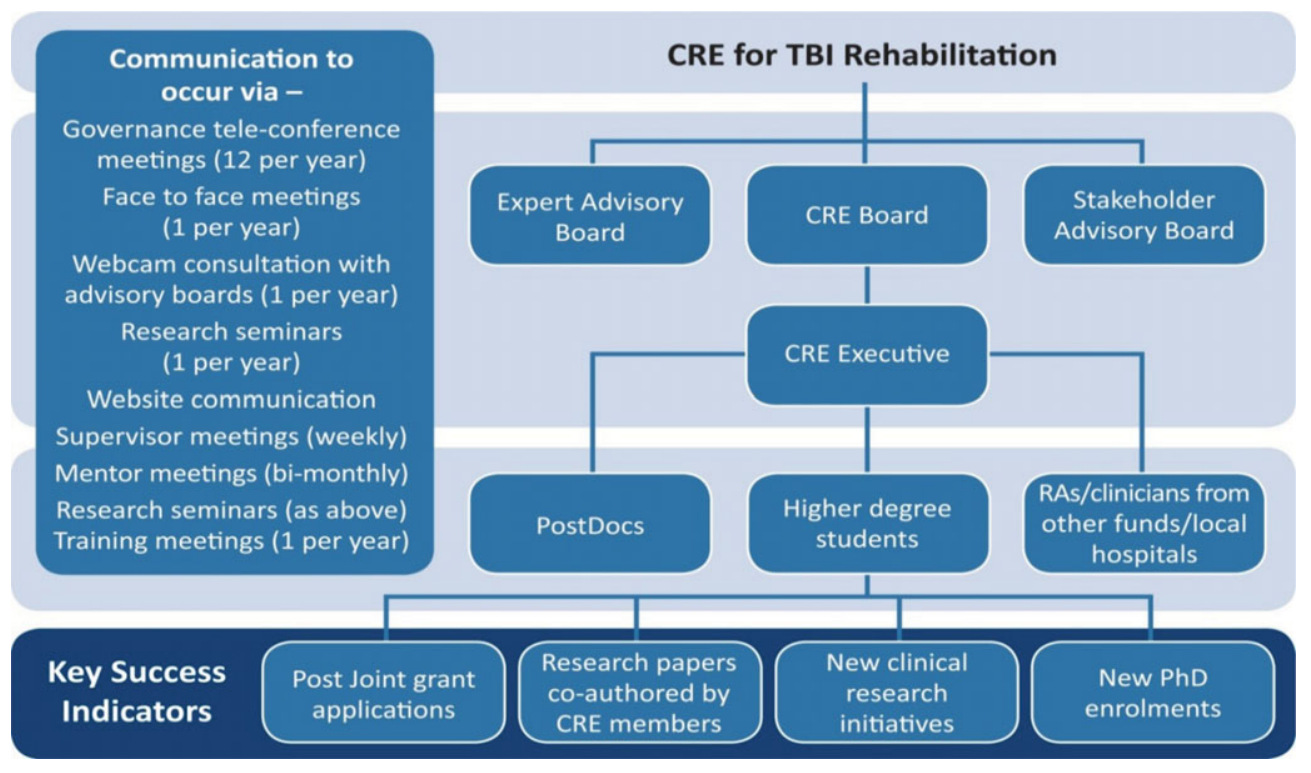

\section{FIGURE 4}

(Colour online) Governance of Moving Ahead and Key Success Indicators of increased collaboration and research training.

input into the research priorities of the CRE and facilitate translation of research into practice. The structure of governance of the CRE is depicted in Figure 4. The CRE Board, comprising Chief and Associate Investigators will make decisions regarding the prioritisation and implementation of research and funds. The Board of Management will be involved in training and supervision of doctoral and postdoctoral students. To maximise skill sharing, funds are allocated for students to travel across sites, to learn new skills and to provide opportunities to work collaboratively.

Communication occurs via monthly governance tele-meetings and one face-to-face meeting organised around training meetings and/or at ASSBI annual conferences. The Moving Ahead Executive meet in person or by teleconference more frequently, as required, and communicates with the CRE Board between meetings. The website plays a pivotal role in the dissemination and exchange of information. Both the Expert Advisory Boards and the Stakeholder Advisory Board are to meet annually with the CRE Board via webcam in separate consultative meetings. These meetings will facilitate feedback from the advisory boards on CRE policies and research directions for the coming year. Communication between other members of the CRE occurs via the training and research seminars, cross-facility visits and mentoring.

\section{Conclusions}

Moving Ahead provides a model for a Centre of Research Excellence in TBI Rehabilitation that is unique in the world. It represents a significant concentration of talent in this field, creating the potential for major gains in understanding the social consequences of TBI and ways of ameliorating these for the benefit of people with TBI and their families. It provides the opportunity for researchers currently working in different domains (education, research, service provision) to share skills in specialist technologies and remediation techniques, to combine research approaches, expand data collection and establish collaborative databases and build work-force capacity. TBI is a major source of disability and new, innovative inroads are needed. Finally, we believe we are Moving Ahead. www.moving-ahead.com. $\mathrm{au}$

\section{References}

Access Economics (2009). The economic cost of spinal cord injury and traumatic brain injury in Australia: A report for the Victorian Neurotrauma Initiative, Canberra: Access Economics, Pty Ltd.

Alderman, N. (2003). Contemporary approaches to the management of irritability and aggression following traumatic brain injury. Neuropsychological Rehabilitation, 13, 211-240. 
Babbage, D.R., Yim, J., Zupan, B., Neumann, D., Tomita, M.R., \& Willer, B. (2011). Meta-analysis of facial affect recognition difficulties after traumatic brain injury. Neuropsychology, 25(3), 277-285.

Beauchamp, M., \& Anderson, V. (2010). SOCIAL: An integrative framework for the development of social skills. Psychological Bulletin, 136, 39-64.

Behn, N., Togher, L., Power, E., \& Heard, R. (in press). Evaluating communication training for paid carers of people with traumatic brain injury. Brain Injury.

Brooks, N., Campsie, L., Symington, C., Beattie, A., \& McKinlay, W. (1987). The effects of severe head injury on patient and relative within seven years of injury. Journal of Head Trauma Rehabilitation, 2(3), $1-13$.

Burke, B.L., Dunn, C.W., Atkins, D.C., \& Phelps, J.S. (2004). The emerging evidence base for Motivational Interviewing: A meta-analytic and qualitative Inquiry. Journal of Cognitive Psychotherapy, 18(4), 309-322.

Cahill, L.M., Murdoch, B.E., \& Theodoros, D.G. (2002). Perceptual analysis of speech following traumatic brain injury in childhood. Brain Injury, 16, 415-446.

Caldwell, E., Whitehead, M., Fleming, J., \& Moes, L. (2008). Evidence-based practice in everyday clinical practice: strategies for change in a tertiary Occupational Therapy Department. Australian Occupational Therapy Journal, 55, 79-84.

Chambless, D.L. (1993). Task force on promotion and dissemination of psychological procedures: A report adopted by the Division 12 Board-October 1993.

Cicerone, K.D., Langenbahn, D.M., Braden, C., Malec, J.F., Kalmar, K., Fraas, M., . . Ashman, T. (2011). Evidence-based cognitive rehabilitation: Updated review of the literature from 2003 through 2008. Archives of Physical Medicine and Rehabilitation, 92, 519-530.

Croker, V., \& McDonald, S. (2005). Recognition of emotion from facial expression following traumatic brain injury. Brain Injury, 19, 787-789.

Cullen, N., Chundamala, J., Bayley, M., \& Jutai, J. (2007). The efficacy of acquired brain injury rehabilitation. Brain Injury, 21, 113-132.

Dahlberg, C., Hawley, L., Morey, C., Newman, J., Cusick, C.P., \& Harrison-Felix, C. (2006). Social communication skills in persons with post-acute traumatic brain injury: Three perspectives. Brain Injury, 20(4), 425-435.

de Sousa, A., McDonald, S., Rushby, J., Li, S., Dimoska, A., \& James, C. (2011). Understanding deficits in empathy after traumatic brain injury: The role of affective responsivity. Cortex, 47(5), 526-535.

Demakis, G.J., Hammond, F., Knotts, A., Cooper, D.B., Clement, P., Kennedy, J., \& Sawyer, T. (2007). The Personality Assessment Inventory in individuals with traumatic brain injury. Archives of Clinical Neuropsychology, 22(1), 123-130.

Denmark, J., \& Geneinhardt, M. (2002). Anger and its management for survivors of acquired brain injury. Brain Injury, 16, 91-108.
Doctor, J.N., Castro, J., Temkin, N.R., Fraser, R.T., Machamer, J.E., \& Dikmen, S.S. (2005). Workers' risk of unemployment after traumatic brain injury: A normed comparison. Journal of the International Neuropsychological Society, 11(6), 747-752.

Fleming, J.M., Strong, J., \& Ashton, R. (1998). Cluster analysis of self-awareness levels in adults with traumatic brain injury and relationship to outcome. Journal of Head Trauma Rehabilitation, 13, 39-51.

Gomez-Hernandez, R., Max, J.E., Kosier, T., Paradiso, S., \& Robinson, R.G. (1997). Social impairment and depression after traumatic brain injury. Archives of Physical Medicine Rehabilitation, 78, 1321-1326.

Hsieh, M., Ponsford, J., Wong, D., Schönberger, M., Taffe, J., \& McKay, A. (2012). Motivational Interviewing and Cognitive Behavior Therapy for Anxiety Following Traumatic Brain Injury: A pilot randomized controlled trial. Neuropsychological Rehabilitation, published online, doi:10.1080/09602011.2012.678860.

Hyder, A.A. (2007). The impact of traumatic brain injuries: A global perspective. NeuroRehabilitation, 22, 341-353.

Jorge, R.E., Robinson, R.G., Moser, D., Tateno, A., Crespo-Facorro, B., \& Arndt, S. (2004). Major depression following traumatic brain injury. Archives of General Psychiatry, 61, 42-50.

Kangas, M., \& McDonald, S. (2011). Is it time to act? The potential of acceptance and commitment therapy for psychological problems following acquired brain injury. Neuropsychological Rehabilitation, 21(2), 250-276.

Kant, R., Duffy, J.D., \& Pivovarnik, A. (1998). Prevalence of apathy following head injury. Brain Injury, 12(1), 87-92.

Kim, S.H., Manes, F., Kosier, T., Baruah, S., \& Robinson, R.G. (1999). Irritability following traumatic brain injury. Journal of Nervous and Mental Disease, 187, 327-335.

Kinsella, G., Packer, S., \& Olver, J. (1991). Maternal reporting of behaviour following very severe blunt head injury. Journal of Neurology, Neurosurgery and Psychiatry, 54(5), 422-426.

Kleim, J.A., \& Jones, T.A. (2008). Principles of experience-dependent neural plasticity: Implications for rehabilitation after brain damage. Journal of Speech, Language, and Hearing Research, 51(1), S225-S239.

Lane-Brown, A.T., \& Tate, R.L. (2008). Interventions for apathy after traumatic brain injury. The Cochrane Database of Systematic Reviews, Issue 2.

Lane-Brown, A.T., \& Tate, R.L. (2009). Measuring apathy after traumatic brain injury: Psychometric properties of the Apathy Evaluation Scale and the Frontal Systems Behavior Scale. Brain Injury, 23, 9991007.

Larson, M.J., Kaufman, D., Schmalfuss, I.M., \& Perlstein, W.M. (2007). Performance monitoring, error processing, and evaluative control following 
severe TBI. Journal of the International Neuropsychological Society, 13, 961-971.

McCabe, P., Lippert, C., Weiser, M., Hilditch, M., Hartridge, C., \& Villamere, J. (2007). Community reintegration following acquired brain injury. Brain Injury, 21, 231-257.

McDonald, S., Li, S., De Sousa, A., Rushby, J., Dimoska, A., James, C., \& Tate, R.L. (2011). Impaired mimicry response to angry faces following severe traumatic brain injury. Journal of Clinical and Experimental Neuropsychology, 33(1), 17-29.

McDonald, S., \& Saunders, J.C. (2005). Differential impairment in recognition of emotion across different media in people with severe traumatic brain injury. Journal of the International Neuropsychological Society, 11(4), 392-399.

McGinn, L., \& Sanderson, W.C. (2001). What allows Cognitive Behavioural Therapy to be brief: Overview, efficacy and crucial factors facilitating brief treatment. Clinical Psychology: Science and Practice, 8, 23-27.

Mealings, M., \& Douglas, J. (2010). 'School's a big part of your life...': Adolescent perspectives of their school participation following Traumatic Brain Injury. Brain Impairment, 11(1), 1-16.

Mealings, M., Douglas, J., \& Olver, J. (2012). Considering the student perspective in returning to school after TBI: a literature review. Brain Injury, 26(10), 1165-1176.

Medd, J., \& Tate, R.L. (2000). Evaluation of an anger management therapy programme following acquired brain injury: A preliminary study. Neuropsychological Rehabilitation, 10, 185-201.

Metcalfe, C., Lewin, R., Wisher, S., Perry, S., Bannigan, K., \& Moffett, J.K. (2001). Barriers to implementing the evidence base in four NHS therapies. Dieticians, occupational therapists, physiotherapists, speech and language therapists. Physiotherapy, 87, 433441.

Miller, W.R., \& Rollnick, S. (2002). Motivational Interviewing: Preparing people for change (2nd ed.). New York: The Guilford Press.

Murdoch, B., Ng, M.L., \& Barwood, C. (2012). Treatment of articulatory dyfunction in Parkinson's disease using repetative transcranial magnetic stimulation. European Journal of Neurology, 19(2), 340347.

Oddy, M., Coughlan, T., Tyerman, A., \& Jenkins, D. (1985). Social adjustment after closed head injury: a further follow-up seven years after injury. Journal of Neurology, Neurosurgery and Psychiatry, 48(6), 564-568.

Olver, J.H., Ponsford, J.L., \& Curran, C.A. (1996). Outcome following traumatic brain injury: A comparison between 2 and 5 years after injury. Brain Injury, 10(11), 841-848.

Ownsworth, T., \& Clare, L. (2006). The association between awareness deficits and rehabilitation outcome following acquired brain injury. Clinical Psychology Review, 26, 783-795.
Ownsworth, T., Fleming, J., Desbois, J., Strong, J., \& Kuipers, P. (2006). A metacognitive contextual intervention to enhance error awareness and functional outcome following traumatic brain injury: A singlecase experimental design. Journal of the International Neuropsychological Society, 12(1), 54-63.

Ownsworth, T., Fleming, J., Strong, J., Radel, M., Chan, W., \& Clare, L. (2007). Awareness typologies, longterm emotional adjustment and psychosocial outcomes following acquired brain injury. Neuropsychological Rehabilitation, 17, 129-150.

Perdices, M., Schultz, R., Tate, R., McDonald, S., Togher, L., Savage, S., ... Smith, K. (2006). The evidence base of neuropsychological rehabilitation in acquired brain impairment (ABI): How good is the research? Brain Impairment, 7, 119-132.

Ponsford, J.L., Olver, J.H., Curran, C., \& Ng, K. (1995). Prediction of employment status two years after traumatic brain injury. Brain Injury, 9, 11-20.

Rapee, R.M., Lyneham, H., Schniering, C., Wuthrich, V., Abbott, M., Hudson, J., \& Wignall, A. (2006). The Cool Kids Child and Adolescent Anxiety Program therapist manual. Sydney: Centre for Emotional Health, Macquarie University.

Robertson, I.H., \& Murre, J.M.J. (1999). Rehabilitation of brain damage: Brain plasticity and principles of guided recovery. Psychological Bulletin, 125(5), 544-575.

Shorland, J., \& Douglas, J. (2010). Understanding the role of communication in maintaining and forming friendships following traumatic brain injury. Brain Injury, 24(4), 569-580.

Soo, C., Tate, R., \& Rapee, R. (2012). Social anxiety and its treatment in children and adolescents with acquired brain injury. In V. A. Anderson \& M. Beauchamp (Eds.), Developmental social neuroscience: Contributions to childhood brain disorders. Sydney: Guilford Press.

Tate, R.L., Broe, G.A., Cameron, I.D., Hodgkinson, A.E., \& Soo, C.A. (2005). Pre-injury, injury and early post-injury predictors of long-term functional and psychosocial recovery after severe traumatic brain injury. Brain Impairment, 6, 75-89.

Tate, R.L., Harris, R.D., Cameron, I.D., Myles, B.M., Winstanley, J.B., Hodgkinson, A.E., ... Harradine, P.G. (2006). Recovery of impairments after severe traumatic brain injury: findings from a prospective, multicentre study. Brain Impairment, 7, 1-15.

Tate, R.L., Lulham, J., Broe, G.A., Strettles, B., \& Pfaff, A. (1989). Psychosocial outcome for the survivors of severe blunt head injury: The results from a consecutive series of 100 patients. Journal of Neurology, Neurosurgery and Psychiatry, 52, 1128-1134.

Tate, R.L., McDonald, S., Simpson, G.K., Lane-Brown, A., Schultz, R., Dunne, R., \& Wilson, B.A (2009). Efficacy of two therapeutic procedures to increase community participation after traumatic brain injury: a randomized controlled trial. Presented at the Neuropsychological Rehabilitation Symposium, Tallinn, Estonia [abstract]. Brain Impairment, 10, 228-229. 
Thurman, D. (1999). Traumatic brain injury in the United States: A public health perspective. Journal of Head Trauma Rehabilitation, 14(6), 602-615.

Togher, L., McDonald, S., Tate, R.L., Power, E., \& Rietdijk, R. (2009). Training communication partners of people with traumatic brain injury: Reporting the protocol for a clinical trial. Brain Impairment, 10, 118-204.

Togher, L., Power, E., Rietdijk, R., McDonald, S., \& Tate, R. (2012). An exploration of participant experience of a communication training program for people with traumatic brain injury and their communication partners. Disability and Rehabilitation, 34(18), 1562-1574.

Toglia, J., \& Kirk, U. (2000). Understanding awareness deficits following brain injury. Neurorehabilitation, $15,57-70$.

Westra, H.A., \& Dozois, D.J. (2006). Preparing clients for cognitive behavioral therapy: A randomized pilot study of motivational interviewing for anxiety. Cognitive Therapy and Research, 30(4), 481-498.

Whelan-Goodinson, R., Ponsford, J., \& Schönberger, M. (2008). The association between psychiatric state and outcome following traumatic brain injury. Journal of Rehabilitation Medicine, 40(10), 850-857.

Whyte, J., \& Hart, T. (2003). Its more than a black box, its a Russian doll: defining rehabilitation treatments.
American Journal of Physical Medicine and Rehabiliation, 82, 639-652.

Woods, D.T., Catroppa, C., \& Anderson, V.A. (2012). Family-centered and parent-based models for treating socio-behavioral problems in children with acquired brain injury. In V.A. Anderson \& M. Beauchamp (Eds.), Developmental social neuroscience: Contributions to childhood brain disorders. Sydney: Guilford Press.

Woods, D.T., Catroppa, C., Barnett, P., \& Anderson, V.A. (2011). Parental disciplinary practices following acquired brain injury in children. Developmental Neurorehabilitation, 14(5), 274-282.

Woods, D., Catroppa, C., Godfrey, C., Giallo, R., Mathhews, J. \& Anderson, V. (under review). Longterm maintenance of treatment effects following intervention for families with children who have acquired brain injury.

Ylvisaker, M., Turkstra, L.S., \& Coelho, C.A. (2005). Behavioral and social interventions for individuals with traumatic brain injury: A summary of the research with clinical implications. Seminars in Speech and Language, 26(4), 256-267.

Young, J.M., Glasziou, P., \& Ward, J.E. (2002). General practitioners' self ratings of skills in evidence based medicine: validation study. British Medical Journal, 324, 950-951. 